NASA Technical Memorandum 101404

AIAA-89-0406

\title{
Hardware Development for the Surface Tension Driven Convection Experiment Aboard the USML-1 Spacelab Mission
}

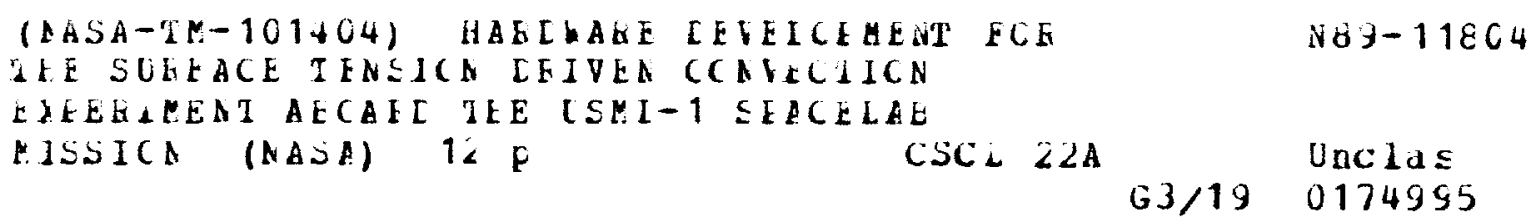

A.D. Pline, T.P. Jacobson, J.S. Wanhainen, and D.A. Petrarca

Lewis Research Center

Cleveland, Ohio

Prepared for the

27th Aerospace Sciences Meeting

sponsored by the American Institute of Aeronautics and Astronautics

Reno, Nevada, January 9-12, 1989 


\title{
HARDWARE DEVELOPMENT FOR THE SURFACE TENSION DRIVEN CONVECTION EXPERIMENT ABOARD THE USML-1 SPACELAB MISSION
}

\author{
A. D. Pline, T. P. Jacobson, J. S. Wanhainen, and D. A. Petrarca \\ NASA Lewis Research Center \\ Cleveland, Ohio 44135
}

\begin{abstract}
The Surface Tension Driven Convection Experiment is a Space Transportation System flight experiment to study both transient and steady thermocapillary fluid flows aboard the USML-1 Spacelab mission planned for March 1992. Hardware is under development to establish the experimental conditions and perform the specified measurements, for both ground-based research and the flight experiment in a Spacelab single rack. Major development areas include an infrared thermal imaging system for surface temperature measurement, a $\mathrm{CO}_{2}$ laser and control system for surface heating, and for flow visualization a He-Ne laser and optical system in conjunction with an intensified video camera. For ground-based work the components of each system have been individually purchased or designed, and tested. The three systems will be interfaced with the balance of the experimental hardware and will constitute a working engineering model. A description of the three systems and examples of the component performance is given along with the plans for the development of flight hardware.
\end{abstract}

\section{$\underline{\text { Introduction }}$}

Access to the low-gravity environment aboard the Space Shuttle, offers unique opportunities for both scientific research and commercial ventures, for example, containerless processing of materials. The effects of natural convection are minimized and contact with a processing crucible may be eliminated in this environment. By removing two of the main sources of imperfections in terrestrial materials processing, the possibility of growing better quality crystals by containerless methods is becoming a reality.

Because liquid/gas free surfaces are inherent to containerless processes, surface tension driven flows in reduced gravity become significant. ${ }^{1}$ Surface tension driven flows, or thermocapillary flows, are driven by a thermally induced surface tension variation along a liquid free surface. This surface tension gradient, established by heating the surface, is a surface tractive force which creates a flow parallel to the liquid free surface from regions of low surface tension to high surface tension. Under certain conditions thermocapillary flow becomes oscillatory ${ }^{2}$ and may detrimentally effect the growth of crystals. Therefore, an understanding of the basic science of both steady state and oscillatory thermocapillary flows is needed in order to realize any benefit from low-gravity materials processing.

As discussed by Ostrach and Kamotani, 3,4 thermocapillary flows, both steady state and oscillatory, have been demonstrated in space (Napolitano et al. ${ }^{5}$ and Schwabe et al. ${ }^{6}$ ). In order to study these phenomenon in a more quantitative fashion, Kamotani et al. ${ }^{3}$ described the considerations and parameters which influence the design of a thermocapillary flow experiment in reduced gravity, resulting in the design of the Surface Tension Driven Convection Experiment (STDCE).

This paper describes the development of three major systems which will be used to establish the experimental conditions and perform some of the desired measurements: an infrared imaging system to measure fluid surface temperatures, a $\mathrm{CO}_{2}$ laser and control system for surface heating, and a He-Ne laser and optical system in conjunction with an intensified video camera for flow visualization. These major systems will be integrated with other systems and will comprise a working engineering model of the experiment. At the present time plans are in progress to procure flight versions of these four components, while all other hardware is under design at the NASA Lewis Research Center (LeRC).

\section{STDCE Overview}

The two experimental configurations for the STDCE are illustrated in Figure 1: a constant temperature boundary condition (called CT experiment), and a constant flux boundary condition (called CF experiment). Two configurations are studied for several reasons. In the $C T$ experiment the total temperature 


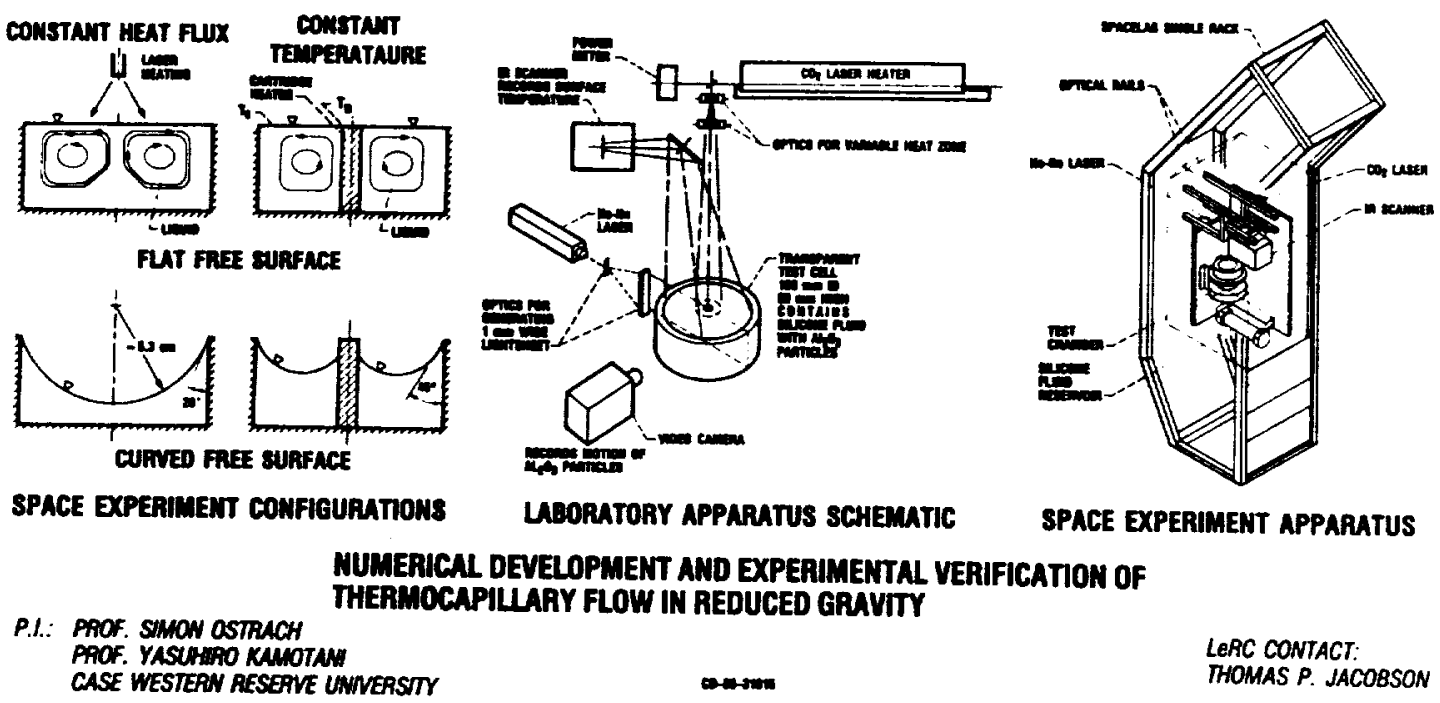

Figure 1. The Surface Tension Driven Convection Experiment.

difference along the free surface is imposed by heating and cooling opposing walls. This temperature difference is related to the magnitude of the driving force $^{7}$ and can be directly measured. In the $C F$ experiment various heat flux distributions are imposed along the free surface, using a radiant heater, while their effect on the flow is studied. 8

Both experimental configurations use a cylindrical container of $10 \mathrm{~cm}$ diameter and $5 \mathrm{~cm}$ in height. The STDCE is conducted in two discrete segments. For the CT experiment a $1.1 \mathrm{~cm}$ diameter by $5 \mathrm{~cm}$ high resistance heater (heated wall) is placed in the center of the test cell (cooled wall). For the CF experiment the resistance heater is removed and the free surface is heated using a radiant heater. For both $C T$ and $C F$ experiments the test container is filled to different levels in order to create both flat and curved free surfaces. Because the thermocapillary driving force acts tangentially to the local free surface, variations in free surface shape permit different driving forces to be used as an experimental parameter. A flat free surface greatly simplifies both the theoretical and experimental analyses and therefore will be used for most of the experiments. A curved free surface, which is unique to a low gravity environment, allows the investigation of a driving force which can not be studied in a terrestrial environment. Consequently, verification of experimental techniques described herein, in conjunction with a curved free surface, is not included. Test matrices which include temperature gradient $(\Delta \mathrm{T})$, Marangoni number $(\mathrm{Ma})^{7}$, heater power, free surface shape and duration for both CT and CF experiments are presented in tables 1 and 2. A more detailed explanation of other experimental considerations and parameters is given in the litcrature. $1,3,6,7$

The experimental hardware for the STDCE is presently in a design phase. The test chamber is made of copper, to insure good thermal boundary conditions at the side wall. A $10 \mathrm{cSt}$ silicone oil is used as the test fluid because it is insensitive to surface 
contamination (a common problem with surface tension experiments), is safe and its viscosity represents the best compromise between flow speed and susceptibility to free surface disturbances.

A planar cross section is illuminated using a $1 \mathrm{~mm}$ wide $\mathrm{He}-\mathrm{Ne}$ laser light sheet which is reflected by seed particles in the fluid, allowing observation of the flows. Because this geometry produces an axisymmetric flow, illumination of only one cross section is needed. The flow data are observed using an intensified video camera, which views the light sheet through a view port in the copper chamber, and are recorded using a video cassette recorder for post-flight analysis.

Bulk flow temperatures are measured using temperature probes placed in the fluid. The heating systems used are a resistance heater (CT experiment) and a $\mathrm{CO}_{2}$ laser (CF experiment). $A$ thermographic technique is used to measure the surface temperature. The imager output is also recorded on videotape and temperature distributions are determined from the thermographs after flight. This hardware is configured to occupy a complete Spacelab single rack.

\section{Science Requirements}

The hardware described in this paper was developed in response to the science requirements developed by the Principal and Co-Investigators (S. Ostrach and $Y$. Kamotani of Case Westem Reserve University). These requirements are necessary to define and guide the development of the experimental hardware. An outline of the requirements for the systems described herein is presented here while the requirements for the experiment in its entirety are given in the Science Requirements Document for the Surface Tension Driven Convection Experiment in Reduced Gravity. 8

Table 1. Constant Flux Tests

\begin{tabular}{lllllll}
\hline Test \# & $\begin{array}{l}\text { Heating } \\
\text { Zone } \\
\text { Dia. }(\mathrm{mm})\end{array}$ & $\begin{array}{l}\text { Total } \\
\text { Power } \\
(\mathrm{W})\end{array}$ & $\begin{array}{l}\Delta \mathrm{T} \\
\left({ }^{\circ} \mathrm{C}\right)\end{array}$ & Ma & $\begin{array}{l}\text { Free Surface } \\
\text { Shape }\end{array}$ & $\begin{array}{l}\text { Duration } \\
(\mathrm{min})\end{array}$ \\
\hline 1 & 10 & 0.5 & 10.4 & $4.2 \times 10^{4}$ & flat & 60 \\
2 & 10 & 3.0 & 36.8 & $1.5 \times 10^{5}$ & flat & 10 \\
3 & 30 & 3.0 & 12.4 & $5.0 \times 10^{4}$ & flat & 10 \\
4 & 5 & 0.2 & 10.2 & $4.1 \times 10^{4}$ & flat & 10 \\
5 & 5 & 3.0 & 64.7 & $4.4 \times 10^{5}$ & flat & 10 \\
6 & 5 & 3.0 & $\approx 64.7$ & $\approx 2.2 \times 10^{5}$ & curved & 10 \\
7 & 30 & 3.0 & $\approx 12.4$ & $\approx 2.5 \times 10^{4}$ & curved & \\
\hline
\end{tabular}

The values of $\Delta \mathrm{T}$ and $\mathrm{Ma}$ for the flat surface tests are computed by numerical analysis and those for the curved surface tests are estimates and $\mathrm{Ma}$ is based on average liquid depth.

Table 2. Constant Temperature Tests

\begin{tabular}{rlllll}
\hline Test \# & $\begin{array}{l}\text { Heater } \\
\text { Power }(\mathrm{W})\end{array}$ & $\begin{array}{l}\Delta \mathrm{T} \\
\left({ }^{\circ} \mathrm{C}\right)\end{array}$ & Ma & $\begin{array}{l}\text { Free Surface } \\
\text { Shape }\end{array}$ & $\begin{array}{l}\text { Duration } \\
(\mathrm{min})\end{array}$ \\
\hline 8 & 1.5 & 10 & $3.9 \times 10^{4}$ & flat & 60 \\
9 & 6.8 & 25 & $1.2 \times 10^{5}$ & flat & 10 \\
10 & 32.8 & 60 & $4.1 \times 10^{5}$ & flat & 10 \\
11 & $\approx 1.5$ & 10 & $\approx 3.2 \times 10^{4}$ & curved & 10 \\
12 & $\approx 32.8$ & 60 & $\approx 3.3 \times 10^{5}$ & curved & 10 \\
\hline
\end{tabular}

The values of heater power for the flat surface tests are computed numerically and those for the curved surface tests are estimates and $\mathrm{Ma}$ is based on average liquid depth. 


\section{Surface Temperature Measurement}

The requirements for the surface temperature measurement system are for a full field non-intrusive measurement using a thermographic technique. The operational wavelength should be chosen so that the detected radiation comes from a region as close to the surface as possible. The spatial resolution and response time should be less than or equal to $1 \mathrm{~mm}$ and 0.1 second respectively. The accuracy of the instrument should be within $\pm 5 \%$ of the $\Delta \mathrm{T}$ between the center and side wall. To satisfy these requirements the technique should be calibrated against measurement by fine thermocouples as well as numerical analysis. ${ }^{8}$

\section{Surface Heating System}

For the $\mathrm{CF}$ experiments a remote heat source is required. To obtain effective surface heating and to simulate heating of an opaque liquid metal in crystal growth [processes], it is desirable that the radiation is absorbed within a small distance from the surface (about $1 \mathrm{~mm}){ }^{8}$ The accuracy of the output power shall be $\pm 10 \%$ of the specified value and the fluctuation with time of $\pm 5 \%$ with a warm up time of $<1$ minute.

\section{Flow Visualization}

The requirements for two dimensional flow visualization are for a $1 \mathrm{~mm}$ light sheet to illuminate aluminum oxide particles in a full cross section of the test chamber. The particles are to be 1-20 micron size with an optimum concentration. The camera and recording device are to have a spatial resolution of 0.2 $\mathrm{mm}$.

\section{System Design and Performance}

\section{Surface Temperature Measurement System}

The analyses by Ostrach and Kamotani ${ }^{3}, 7$ identify the thermal signature along the free surface as a critical parameter, as it is the driving force for thermocapillary flows. Therefore quantification of the thermal signature is essential for both ground-based research and the flight experiment. This is accomplished using an infrared thermal imaging system (Fig. 2) to perform the measurements in the laboratory. The performance of this system is used as a baseline for a flight qualified version.

In his work with this system Pline ${ }^{9}$ details the step by step process undertaken to understand and calibrate the infrared imager for this application, as outlined in the Science Requirements Document. The considerations examined for this system were the absorption characteristics of silicone oil, effective emissivity of silicone oil, and the agreement of the imager measurements with thermocouple measurements and numerical calculations.

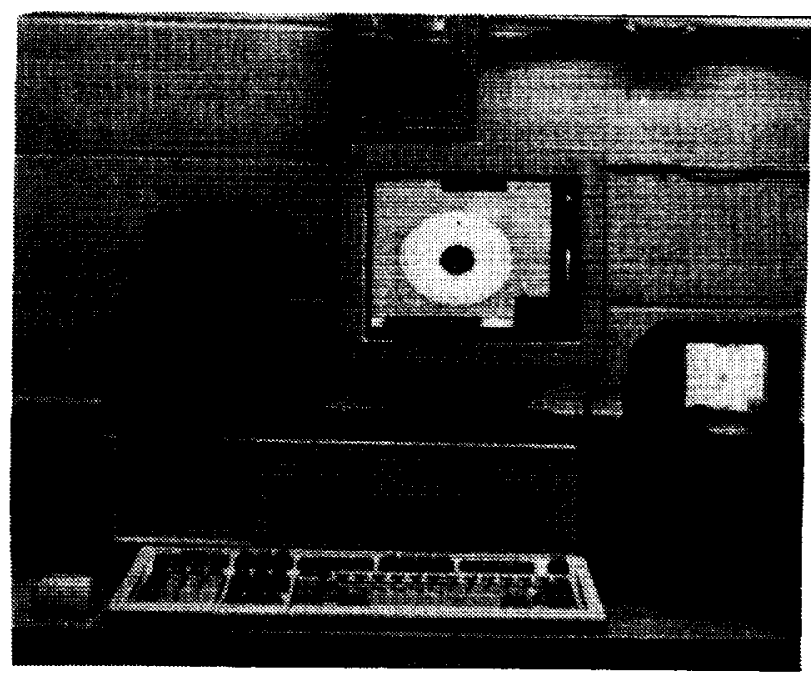

Figure 2. Infrared thermal imaging system.

Absorption Length. The absorption characteristics of silicone oil must be known to understand the thickness of the "surface" as seen by the imager. For semi-transparent targets, radiation is emitted (absorbed) over varying depths depending on the radiation wavelength and material properties. If this depth, usually referred to as the mean absorption length, $1 / k$, where $\mathrm{k}$ is the extinction coefficient, is much less than the total fluid thickness the material is said to be opaque. As a result the measurement of the emitted radiation is considered to be from the "surface". Physically, the mean absorption length represents the fluid thickness which attenuates the incident radiation by a factor of $1 / \mathrm{e}(37 \%)$.

Results for the mean absorption length for the spectral region, corresponding to the operating wavelength region of the imager, were obtained using an infrared spectrophotometer. The spectrophotometer measures radiation attenuated through a variable thickness of fluid as a function of wavelength. The transmission versus wavelength results show that silicone oil is very transparent under 8 microns. Therefore, an imager that operates in the far infrared 
(>8 micron) is needed. In order to find the mean absorption length the transmission through the fluid is averaged over the operating wavelength of the imager (8-12 micron) and plotted against fluid thickness (Fig. 3). The mean absorption length is found graphically from this plot to be $0.012 \mathrm{~mm}$. Also, the fluid thickness which attenuates nearly $100 \%$ of the incoming radiation is found to be $0.200 \mathrm{~mm}$. From these results it is concluded that the "surface" the imager sees is 0.200 mm thick.

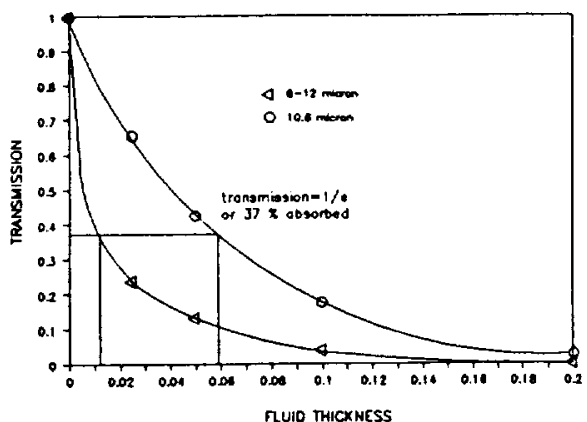

Figure 3. Transmission versus fluid thickness: mean absorption lengths.

Effective Emissivity. Because no surface is an ideal radiator, there is an emissivity $(<1)$ associated with that surface. This emissivity is needed as an input to the thermal imaging system to account for non-ideal radiation and reflected background radiation. There is no existing literature documenting the emissivity of silicone oil. Thus an emissivity is determined empirically. This value is called the effective emissivity because it is implied by comparison of the imager surface temperature data with other techniques, rather than by direct measurement.

Temperature distributions from thermocouples and numerical calculations are compared to temperature distributions from the imager, using different values of the emissivity. From this comparison, surface temperature difference, $T-T_{c}$, where $T_{c}$ is the cooled wall temperature, are plotted against non-dimensional radial position $r / R$, where $R$ is the radius of the test chamber. The emissivity value of 0.90 gives the best agreement with the thermocouple measurements and the calculated surface temperature (Fig. 4). It must be noted that this effective emissivity measurement is specific to this imaging system and these experimental conditions.
Accuracy. Data acquired using the infrared imaging system for both the $C T$ and $C F$ experiments are analyzed and compared to thermocouple measurements and a calculated surface temperature distribution to establish the accuracy of the system.

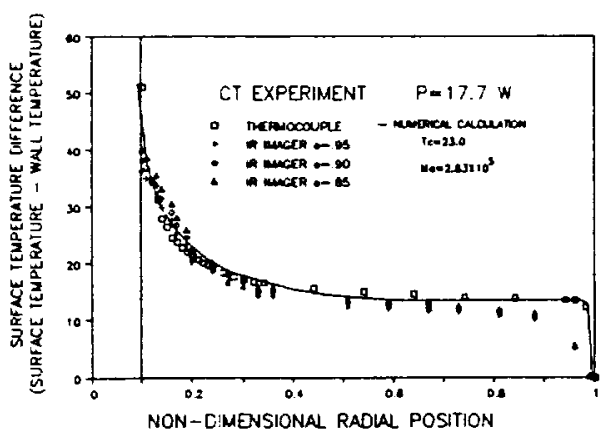

Figure 4. Empirical determination of effective emissivity.

Shown in Figure 5 is the comparison of the three techniques for the maximum temperature gradient expected for the CT experiment (test \#10, Table 2), which is the most difficult CT measurement case. The agreement between the techniques for this case (and a lower power case not shown) is $\pm 5 \%$ of the overall $\Delta \mathrm{T}$ between the heater and the side wall in all but the regions close to the walls. These errors near the walls

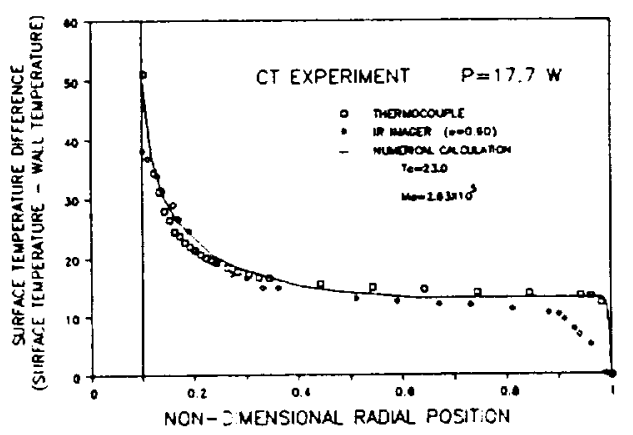

Figure 5. Surface temperature distributions: CT experiment.

are caused by several factors including the imager spatial resolution, low surface emissivity of the wall and a meniscus at the wall. These sources of errors and their possible solutions are discussed elsewhere in detail. 9 
The results shown in Figure 6 are for the most difficult CF measurement case (test \#5, Table 1). The accuracy as compared to the numerical calculation is $\pm 5 \%$ of the $\Delta \mathrm{T}$ except near the wall and in the center of the heating zone. Only the numerical analysis was used for the comparison because thermocouple measurements could not be made with any certainty due to the thin thermal boundary layer under the heating zone and the effect of direct heating of the thermocouple by the $\mathrm{CO}_{2}$ laser. As with the $\mathrm{CT}$ experiment the measurement errors increased near the side wall for reasons stated above. Near the center the errors increased because of the aforementioned boundary layer thickness. The imager reports a lower temperature than the numerical calculation because the imager "sees" through the boundary layer and detects radiation from the cooler fluid beneath the thermal boundary layer.

The plans for obtaining a flight qualified infrared imager are in progress. An imager will be developed, fabricated, tested and qualified for flight. The completion date for the flight version will correspond with the fabrication and integration of all hardware into the complete flight configuration.

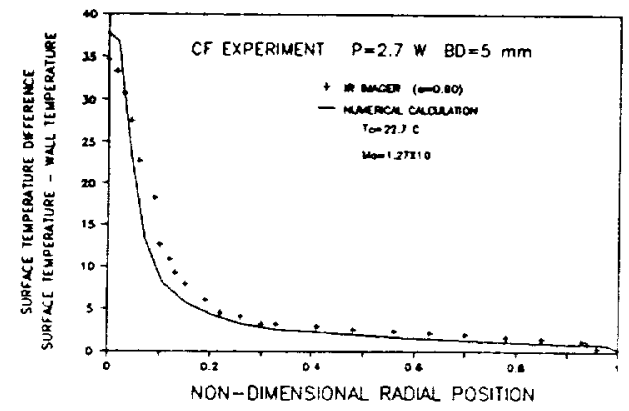

Figure 6. Surface temperature distributions: CF experiment.

\section{Surface Heating System}

Several requirements have driven the surface heating system design towards a remote radiant heater for the $\mathrm{CF}$ experiments. It is necessary to change the radiation signature of the heater for the different test runs and it is desirable, from safety considerations, to locate the source of the radiation away from the free surface.

An RF exited waveguide $\mathrm{CO}_{2}$ laser (Fig. 7), shown with a piggyback low power $\mathrm{He}-\mathrm{Ne}$ laser used for alignment in the laboratory, was chosen as the surface heater for several reasons. It produces radiation at a wavelength which silicone oil readily absorbs $(10.6 \mu)$, the beam diameter is easily varied through a combination of lenses and can be controlled to produce a steady output. These features facilitate meeting the science requirements.

Absorption Length. In addition to the 8-12 $\mu$ region absorption length, an absorption length for the $\mathrm{CO}_{2}$ laser radiation was calculated at $10.6 \mu$. Transmission at $10.6 \mu$ was plotted versus fluid thickness (Fig. 3). From this figure the mean absorption length is 0.060 $\mathrm{mm}$. The fact that the $\mathrm{CO}_{2}$ laser radiation penetrates deeper than the imager sees is advantageous, because if the situation were opposite, the temperature indicated by the imager would be an average over a region where a large temperature gradient exists.

Power Control System Design. To remove waste heat, the $\mathrm{CO}_{2}$ laser is mounted on a water-cooled base plate which removes the heat via conduction. The stability of the output power (both wavelength and amplitude) is dependent on the thermal stability of the laser and will, therefore, be a function of the thermal stability of the base plate. In the laboratory the output power fluctuations measured with the present laser, without a closed loop control system, can be held to within the science requirements by circulating tap water through the base plate. Operation aboard the Shuttle is considerably different due to the temperature fluctuations in the Spacelab cooling system.

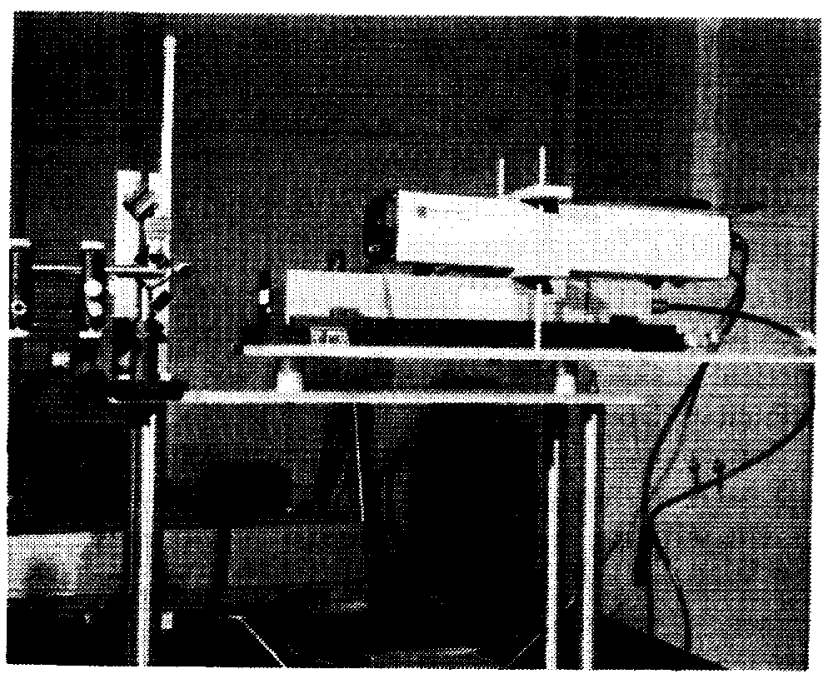

Figure 7. $\mathrm{CO}_{2}$ laser.

Open loop control tests indicate the fluctuations with time, under Spacelab simulated conditions, exceed that 
allowed by the science requirements. Therefore, a closed loop control system was designed by Wenzler and Eichenberg ${ }^{10}$ to control the laser output.

A block diagram of the basic design is shown in Figure 8. The principle of the design is that the laser output is controlled and varied over a small range (3-4 watts) using a closed loop system. The different power levels required (see Table 1) are created using variable ratio beam splitters as turning mirrors to deflect the desired amount of radiation to the free surface. Small changes in set point will be required to compensate for any errors in the beam splitter ratios.

The closed loop operates in the following manner: a set point is entered into a PID controller from the STD bus (the onboard experiment computer) and a control signal, called the trim voltage, is feed to a DC-DC power converter which draws power from the Spacelab power bus. The converter has a gate which is essentially a switch that opens and closes the circuit to start and stop the laser operation. The output of the converter is proportional to the trim voltage regardless of the Spacelab power bus fluctuations. This voltage powers the $\mathrm{CO}_{2}$ laser RF power supply, and in turn regulates the output power of the laser.

As mentioned previously the laser beam is split to the desired ratio with the fraction not reaching the surface, entering a power meter. The power meter output is input to the laser control printed circuit board and is amplified. A provision for adjusting the zero reading is

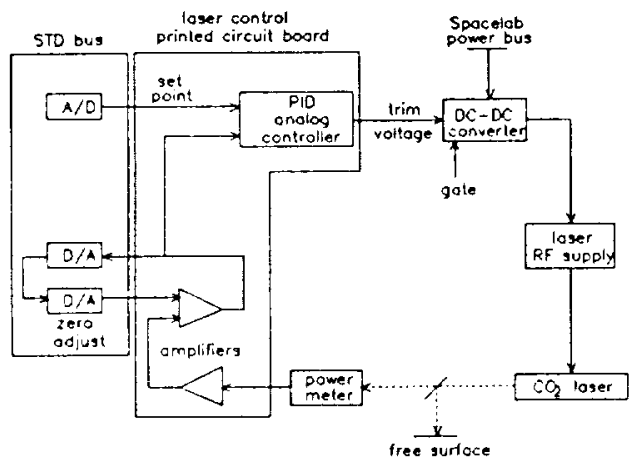

Figure 8. $\mathrm{CO}_{2}$ laser closed loop control system block diagram.

added by measuring the signal with the gate open and nulling it via the STD bus. The signal is then input to the controller, compared to the set point and adjusted accordingly, completing the loop.

Test Results. Both the open loop and closed loop control systems have been tested extensively. The output power fluctuations using the open loop control are within the science requirements (with good thermal control) and exceed the science requirements (with no thermal control). The closed loop control results show that this system is more than adequate to meet the science requirements under all thermal conditions. Figure 9 is a typical plot of the laser output as a function of time, beginning from start up. There is a $12 \%$ overshoot at start up but the output power is within $\pm 2 \%$ of the setpoint after 10 seconds. Tests were run to determine the effects of Spacelab power bus and cooling loop fluctuations. In all cases simulating the Spacelab conditions the output power was similar to that in Figure 9. The system must be calibrated with the optical components (lenses, beam splitters, windows etc.) in place in order to determine accurately the setpoint voltages.

Similar to the IR imager, a $\mathrm{CO}_{2}$ laser will be designed, fabricated, tested and qualified for flight aboard the Shuttle.

\section{Flow Visualization System}

The second major type of data to be returned with the STDCE is a record of the fluid motion. The two main components of this system are a low power

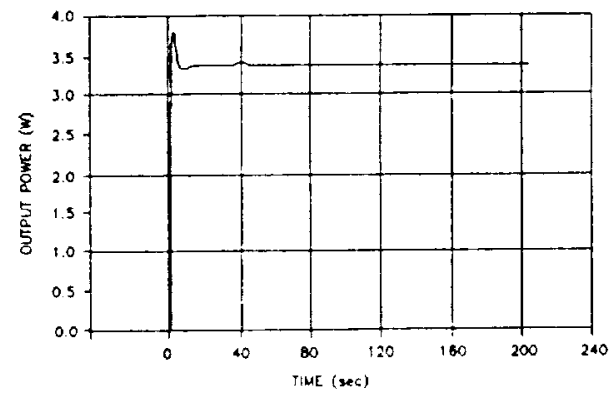

Figure 9. $\mathrm{CO}_{2}$ laser output versus time with closed loop control

$\mathrm{He}-\mathrm{Ne}$ laser and an intensified video camera. The basic concept, outlined above, is to expand a $\mathrm{He}-\mathrm{Ne}$ laser into a sheet of small thickness and illuminate a cross section of the test chamber. Aluminum oxide particles mixed in the fluid reflect the $\mathrm{He}-\mathrm{Ne}$ light which is received by the video camera. This signal is then recorded on a video cassette recorder for later analysis. 
Design Considerations. Several factors contributed to the present design; for example power consumption of the $\mathrm{He}-\mathrm{Ne}$ laser versus light levels for the video camera and optical paths of the $\mathrm{He}-\mathrm{Ne}$ and $\mathrm{CO}_{2}$ laser beams. A major consideration for a Shuttle flight experiment is power consumption. In order to keep the power consumption low, a 5 milliwatt $\mathrm{He}-\mathrm{Ne}$ laser drawing approximately 20 watts was selected as an illumination source. If the flow is to be observed using such a low power source an intensified video camera is needed to detect enough light to produce a usable image, regardless of particle concentration (within the ranges practical for this application). The camera now in use in the laboratory is a silicon intensified tube (SIT) camera. The resolution of this camera is approximately $700 \mathrm{TV}$ lines at light levels of $10^{-2}$ to $10^{-4}$ foot candles providing a spatial resolution better than $0.2 \mathrm{~mm}$. The resolution of the entire system is limited by the recording technique used, S-VHS and $3 / 4^{n}$ formats in the laboratory and on the Spacelab respectively. Therefore the system resolution is that of the recording device (approximately $400 \mathrm{TV}$ lines or approximately $0.25 \mathrm{~mm}$ ). The combination of these two pieces of equipment produces suitable standard TV images for post-flight analysis.

The second consideration is the optical paths of the $\mathrm{CO}_{2}$ and $\mathrm{He}-\mathrm{Ne}$ lasers. In order to illuminate a cross section of the copper chamber using the He-Ne laser expanded into a sheet, the sheet must enter the chamber normal to the free surface. During the $\mathrm{CF}$ experiment the $\mathrm{CO}_{2}$ and $\mathrm{He}-\mathrm{Ne}$ laser beams must be in the same plane. A system was designed to eliminate blocking of either path by turning mirrors. Figures 10 and 11 show simplified front and top views of this design. The light sheet, created using plano-cylindrical lenses, is split into two sheets with prisms, allowing a section of the turning mirror, which reflects the sheet to the surface, to be removed. The two sheets are recombined as they converge on the test section. The $\mathrm{CO}_{2}$ beam is reflected downwards to the surface slightly above the $\mathrm{He}-\mathrm{Ne}$ turning mirror, with the $\mathrm{CO}_{2}$ beam passing through the cut-out section of the $\mathrm{He}-\mathrm{Ne}$ mirror, both beams being in the same plane. A consequence of this design is the superposition of the two He-Ne sheets in the test cell. This creates a single sheet of nearly constant intensity, eliminating the problem of non-uniform intensity caused by the gaussian beam distribution. Figure 12 is an image created using the flow visualization system with a $35 \mathrm{~mm}$ SLR camera, utilizing streak photography, substituted for the SIT camera in order to illustrate the flow structure.

The flight versions of the He-Ne laser and intensified video camera (not necessarily a SIT camera) are the third and fourth major components which will be designed, fabricated, tested and qualified for flight.

Post Flight Data Analysis. The video record of the flow will be analyzed using a computer tracking and motion analysis system. ${ }^{11}, 12$ This system tracks targets by following them from video frame to frame. The targets are outlined based on target/background contrast and digitized, throwing out all information except the digitized outline. A centroid is calculated from the outline and is tracked through a sequence of frames. The frame acquisition rate is selectable to account for variations in flow velocity ranges throughout the test cross section.

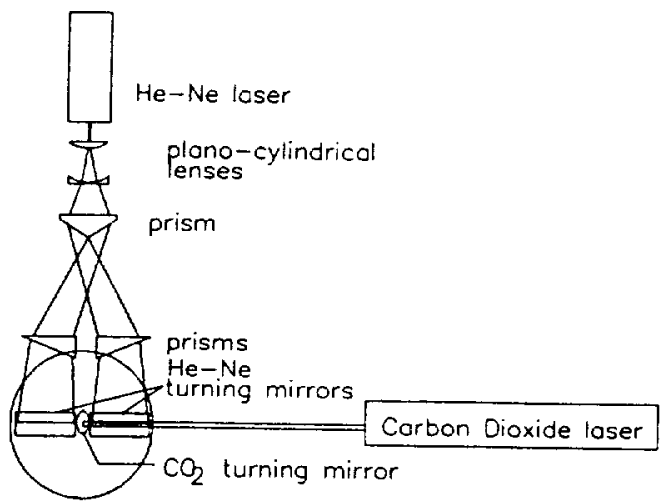

Figure 10. Top view of optical path.

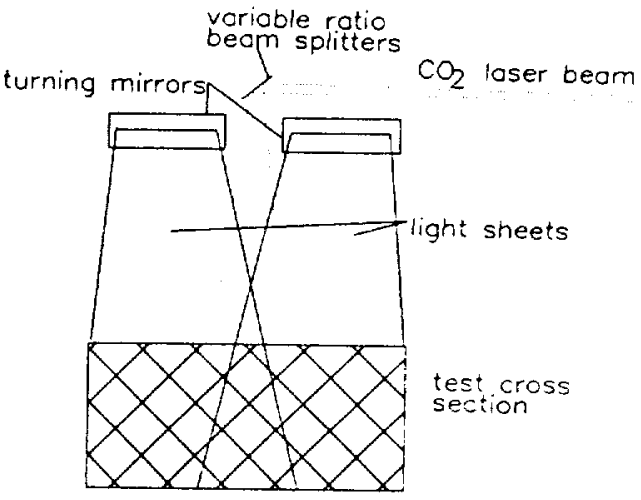

Figure 11. Side view of optical path.

It has been demonstrated that this system is able to track particles illuminated with a 5 milliwatt $\mathrm{He}-\mathrm{Ne}$ laser, imaged with the SIT camera and recorded using a standard VHS system. More work is needed to optimize particle concentration and perform softwarc modifications to display the data in a useful form. 


\section{Concluding Remarks}

The laboratory components described herein, along with the balance of the engineering model hardware presently being designed and fabricated at LeRC, will constitute a working engineering model of the STDCE. For the flight hardware the IR imager, $\mathrm{CO}_{2}$ laser, $\mathrm{He}-\mathrm{Ne}$ laser and intensified video camera will be designed, fabricated, tested, and flight qualified by an outside source, while the balance of the flight hardware will be designed, fabricated, tested and flight qualified at LeRC.

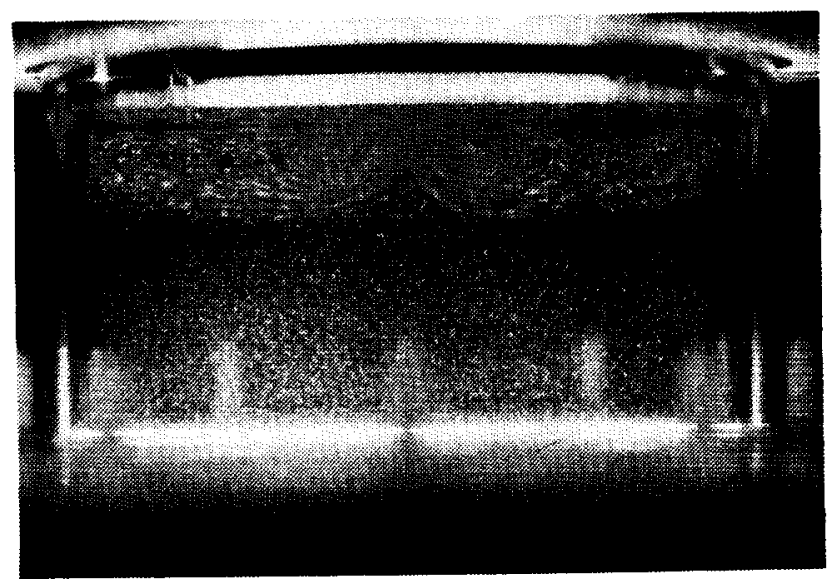

Figure 12. STDCE flow structure.

\section{Acknowledgements}

The authors would like to acknowledge and thank the members of the STDCE design team at the NASA Lewis Research Center for their involvement in and contributions to the design of these systems.

\section{$\underline{\text { References }}$}

1. Ostrach, S.,"Low Gravity Fluid Flows," Annual Review of Fluid Mechanics, Vol. 14, 1982, pp. 313-345

2. Preisser, F., Schwabe, D. and Scharmann, A., "Steady and Oscillatory Thermocapillary Convection in Liquid Columns with Free Cylindrical Surface," Journal of Fluid Mechanics,
Vol. 126, Jan. 1983, pp. 545-567

3. Kamotani, Y. and Ostrach, S., "Design of a Thermocapillary Flow Space Experiment," Journal of Thermophysics and Heat Transfer, Vol. 1, No. 1, 1987, pp. 83-89

4. Ostrach, S., Kamotani, Y. and Lai, C-L., "Oscillatory Thermocapillary Flow," PCH Journal, Vol. 6, No. 5/6, 1985, pp. 585-599

5. Napolitano, L. G., Nonti, R. and Russo, G. "Some Results of the Marangoni Free Convection Experiment, "Proceedings of the 5th European Symposium on Material Sciences under Microgravity, Schloss Elmau, FRG, Nov. 1984, pp. 15-22

6. Schwabe, D., Preisser, F. and Scharmann, A., "Verification of the Oscillatory State of Thermocapillary Convection in a Floating Zone Under Low Gravity," Acta Astronautica, Vol. 9, No. 4,1982 , pp. $265-273$

7. Ostrach, S., "Convection Due to Surface Tension Gradients," in (COSPAR) Space Research, (ed. M.J. Rycroft), 19:563-570, 1979, Oxford/New York:Pergamon

8. Ostrach, S. and Kamotani, Y., "Science Requirements Document for the Surface Tension Driven Convection Experiment in Reduced Gravity," Case Western Reserve University, 1987

9. Pline, A., "Infrared Surface Temperature Measurements for the Surface Tension Driven Convection Experiment," NASA TM 101353, 1988

10. Wenzler, C. and Eichenberg, D.," Design of a $\mathrm{CO}_{2}$ Laser Power Control System for a Spacelab Microgravity Experiment," Report in progress, NASA Lewis Research Research Center, Cleveland, $\mathrm{OH}$.

11. Greaves, J. O. B., "State of the Art in Automated Motion Tracking and Analysis Systems," High Speed Photography, Videography, and Photonics IV, Proc. SPIE 693, pp. 277-281, 1986

12. Walton, J. S., "The Accuracy and Precision of a Video-base Motion Analysis Systcm," High Speed Photography, Videography, and Photonics IV, Proc. SPIE 693, 1986 


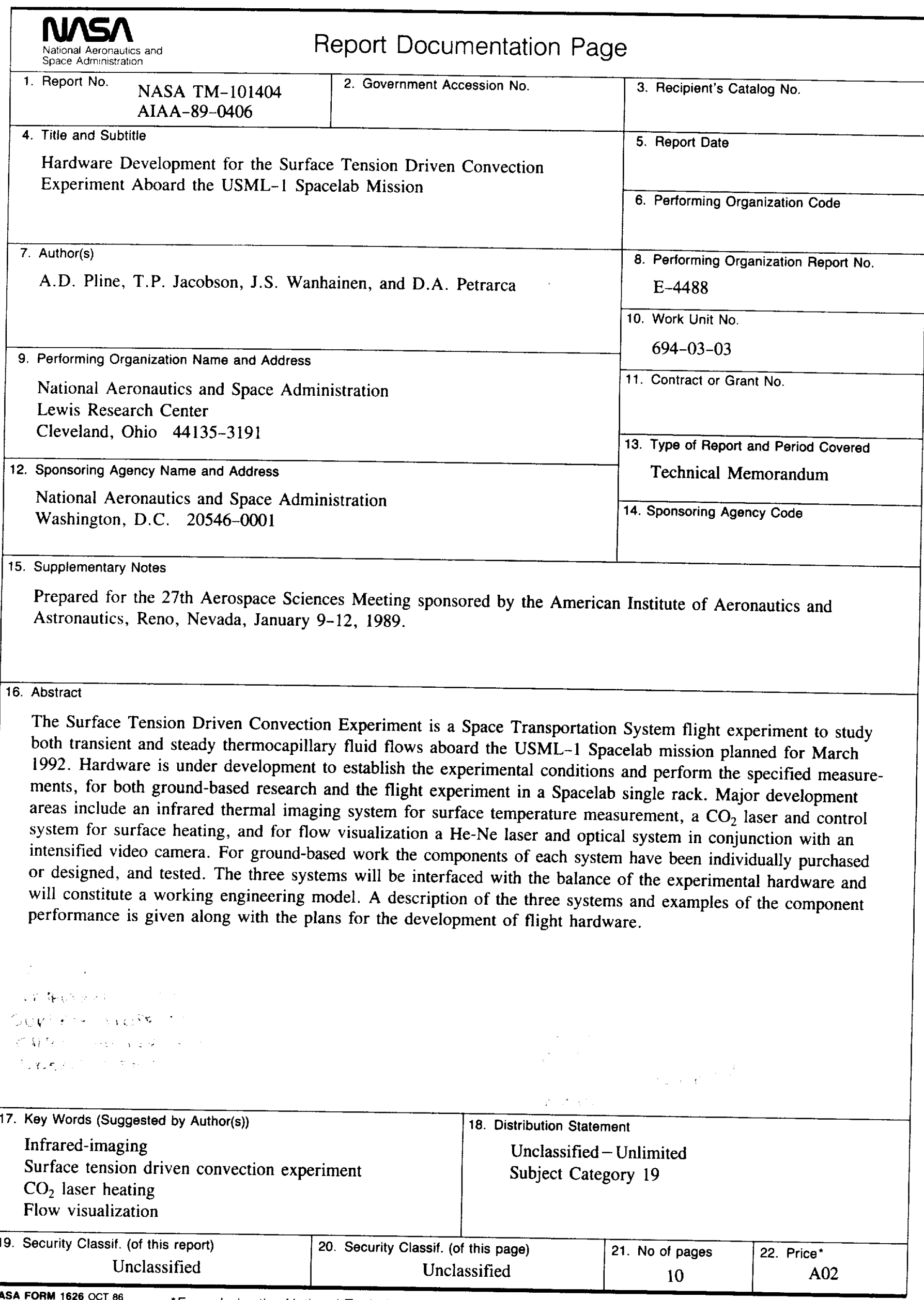

